\title{
Automated assessment of intranasal trigeminal function*
}

\section{T. Hummel, C. Kaehling, F. Grosse}

Smell and Taste Clinic, Department of Otorhinolaryngology, TU Dresden, Germany
Rhinology 54: 27-31, 2016

DOI:10.4193/Rhino15.002

*Received for publication:

January 4, 2015

Accepted: April 29, 2015

\begin{abstract}
Background: The intranasal trigeminal system is a key player in the perception of intranasal airflow. Why it has not been studied very well may be due to the lack of techniques that allow for fast, reliable and inexpensive routine investigation of the system. The basis of the current study is the notion that - within limits - the intranasal trigeminal system detects the overall mass of a stimulus and not just its concentration. Thus, changing the duration of the stimulus at a given concentration has a similar effect as changing its concentration.
\end{abstract}

Methodology: Ninety-nine normosmic subjects participated [48 women and 51 men; mean (range) age $=45$ years (20-88 years)] In addition, 50 patients with olfactory loss were investigated once ( 28 women, 22 men; mean age 58 years, SD = 14 years; age range 24-88 years; causes of olfactory loss: viral infections $n=22$, head trauma $n=8$, chronic sinunasal disease $n=3$, idiopathic $\mathrm{n}=17$ ). $\mathrm{CO}_{2}$-stimuli with various durations (multiples of $50 \mathrm{~ms}$ ) were presented through a standard bilateral nasal cannula at an interval of $10 \mathrm{~s}$; stimulus duration was increased by $50 \mathrm{~ms}$ from one stimulus presentation to the next, until the subject pushed a button indicating a painful sensation. This was the basis for automated assessment of $\mathrm{CO}_{2}$-pain responsiveness.

Results: This current study had four main findings: (1) Using the new, automated device $\mathrm{CO}_{2}$ pain responsiveness can be measured reliably, (2) $\mathrm{CO}_{2}$ pain responsiveness correlates with olfactory function, (3) as with olfaction, women are more sensitive to $\mathrm{CO}_{2}$, and $\mathrm{CO}_{2}$ - pain responsiveness also correlates with aging, (4) $\mathrm{CO}_{2}$ - pain responsiveness is lower in patients with olfactory loss compared to normosmic, healthy controls, even when controlling for age.

Conclusion: We have demonstrated that the current approach is a reliable and valid measure of intranasal trigeminal function.

Key words: smell, nose, olfaction, trigeminal system, $\mathrm{CO}_{2}$

\section{Introduction}

Most odorants stimulate, at least at high concentrations, both the olfactory and the trigeminal nerves ${ }^{(1-3)}$. Thresholds for trigeminal sensations, such as burning, cooling, stinging, and fullness, are generally higher than thresholds for olfactory sensations ${ }^{(4)}$. Clinically, the trigeminal system has received little attention. This is despite the fact that the sensation of airflow is mediated through the trigeminal nerve, which is nicely illustrated with menthol lozenges. When they are sucked on, menthol activates receptors in the respiratory epithelium and, consecutively, the nasal airflow is perceived as stronger. This is, in turn, interpreted as a widening of the nasal cavity, which is not the case
$(5,6)$. In fact, it has been suggested that nasal airflow perception is related to cooling of the nasal mucosa rather than, for example, to obstruction of the nasal passages ${ }^{(7,8)}$. An alternative example is local anesthesia of the nasal cavity that produces a strong feeling of stuffiness. Based on the finding that patients receiving sinus surgery are typically less sensitive to trigeminal stimuli than controls ${ }^{(9,10)}$, it has been speculated that some patients may receive nasal surgery because of a decreased sensation of airflow, not because of a significant nasal congestion.

A number of psychophysical techniques have been introduced to study the function of the nasal trigeminal nerve ${ }^{(11)}$. Among these is the stimulation with $\mathrm{CO}_{2}$ gas, which is thought to be a 
specific trigeminal stimulant that has little or no smell ${ }^{(12)}$. Other techniques involve the lateralized presentation of chemical stimuli with the subjects' task to identify the nostril that had been stimulated. This is based on the idea that lateralization of intranasal chemical stimuli is only possible through the trigeminal nerve ${ }^{((1,4,13-15), \text { but see also }(16))}$.

The basis of the current study is the notion that the trigeminal system detects the overall mass of a stimulus rather than its concentration ${ }^{(17)}$. Thus, changing the duration of the stimulus at a given concentration should have the same effect as changing its concentration. Therefore it should be possible to apply the irritant $\mathrm{CO}_{2}$ at relatively low flows, but with pulses of $100 \%$ concentration and different durations. After building an apparatus that provided these stimuli, we investigated the test-retest reliability of these responses, their relation to age and sex of the subjects, and their clinical applicability in patients with olfactory loss.

\section{Materials and methods}

The study was conducted at the Smell \& Taste Clinic, Department of Otorhinolaryngology of the TU Dresden (protocol number EK156052012). It was approved by the Ethics Committee of the Medical Faculty at the TU Dresden. All chemosensory measurements were carried out in well-ventilated rooms by the same investigator (CK).

\section{Subjects}

Ninety-nine normosmic subjects participated [48 women and 51 men; mean (range) age $=45$ years (20-88 years)]. All of these subjects maintained that they had a normal sense of smell. Olfactory function was assessed by the "Sniffin' Sticks" package which included assessment of phenyl ethyl alcohol odour thresholds, and an extended odour identification test consisting of 32 items ${ }^{(18,19)}$. Normosmia was defined as a test result of 23 and more points on the 32 -item odour identification test ${ }^{(20-22)}$. Exclusion criteria were neurological or rhinological conditions associated with olfactory disorders, including major septal deviations, as assessed with nasal endoscopy and acoustic rhinometry. Subjects were asked to refrain from smoking, eating, or drinking anything other than water for at least one hour prior to testing.

\section{Patients}

In addition, a total of 50 patients with olfactory loss were investigated once ( 28 women, 22 men; mean age 58 years, SD $=14$ years; age range 24-88 years). All patients underwent a structured interview, a detailed otorhinolaryngological examination including nasal endoscopy by a specialized physician, and various tests of gustatory or olfactory function (e.g., "Sniffin' Sticks", taste strips, taste sprays, "Schmeckpulver" for retronasal olfactory testing, olfactory event-related potentials $\left.{ }^{(23)}\right)$. Struc- tural brain imaging using MRI scans was performed whenever deemed necessary. Olfactory loss was caused by viral infections of the upper respiratory tract in $n=22$, by head trauma in $n=8$, by chronic sinunasal disease in 3 patients, and was of idiopathic cause in $n=17$

\section{Trigeminal stimulus presentation device}

The stimuli were delivered by a portable device containing a small $\mathrm{CO}_{2}$ cylinder together with a pressure reducer and a pressure regulator. Stimuli with various durations (multiples of 50 ms) were presented through a standard bilateral nasal cannula; stimulus durations were regulated through a computer-operated valve.

Stimuli were presented at an interval of $10 \mathrm{~s}$ with the subjects being alerted to that by a small signal lighting up $3 \mathrm{~s}$ before stimulus presentation; stimulus duration was increased by 50 ms from one stimulus presentation to the next, until the subject pushed a button - indicating a painful sensation. Then the stimulus duration was reduced until the subject did not push the button anymore. Then the duration was increased again and so forth. The average of the last four turning points of this staircase (calculated by the microcomputer implemented in the stimulation device) was used as an estimate of the $\mathrm{CO}_{2}$ pain threshold, further termed as $\mathrm{CO}_{2}$ "responsiveness". Thus, patients only followed one instruction: "Push the button when you found the stimulus to be painful". In this way, the entire procedure was fully automated. In the present experiment, during each of the two sessions this procedure was repeated three times, and the average of the 3 measures per session was used for further statistical analyses. The entire procedure lasted about $5 \mathrm{~min}$.

\section{Statistical analysis}

SPSS 21.0 for Windows (SPSS Inc., Chicago, IL, USA) was used for statistical analyses. T-tests and analyses of variance (for repeated measures) were used whenever appropriate. For post-hoc testing we used t-tests with Bonferroni-correction for inflated alpha. Pearson correlation coefficients were computed. The alpha-level was set at 0.05 .

\section{Results}

No significant difference was found between trigeminal $\mathrm{CO}_{2}-$ pain responsiveness from sessions 1 and 2 ( $\mathrm{t93}=0.14, \mathrm{p}=0.89$ ). In contrast, there were significant correlations between results from sessions 1 and 2 ( $r 94=0.75, p<0.001)$ (Figure 1 ). With regard to measurements in the control group, women were more sensitive than men (session 1: women: mean \pm SEM $443 \pm 59$ ms, men: $627 \pm 68 \mathrm{~ms}$; $\mathrm{t} 92=2.03, \mathrm{p}=0.045$; session 2: women: $415 \pm 58 \mathrm{~ms}$, men: $645 \pm 72 \mathrm{~ms}$; $\mathrm{t} 92=2.48, \mathrm{p}=0.015$ ). Thresholds increased with age as indicated by significant coefficient of correlations obtained in session 1, but not in session 2 (session 1: $r 94=0.25, p=0.021$; session 2: $r 94=0.12, p=0.27$ ). 

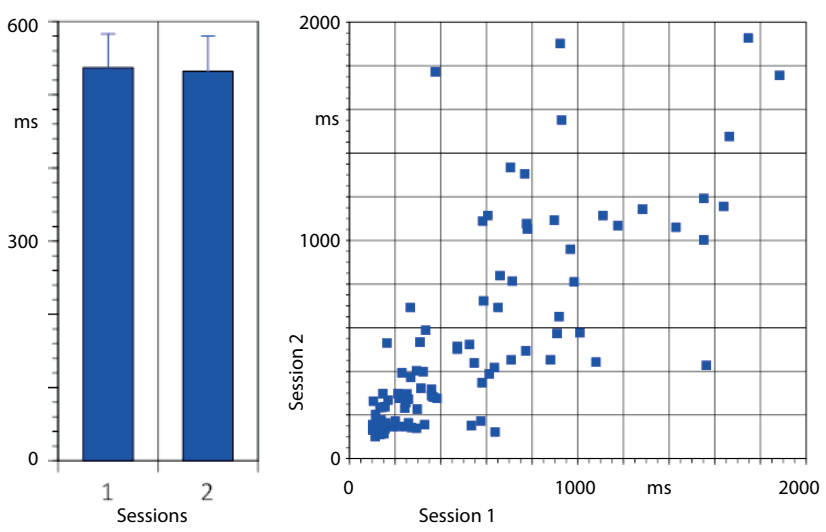

Figure 1. Left: Average pain responsiveness (in $\mathrm{ms}$ ) for the first and second session. Right: Correlation between results from sessions 1 and 2. There was no significant difference between sessions 1 and 2 (the shorter the latency, the more sensitive the subject); results from the two sessions exhibited a good test-retest reliability.

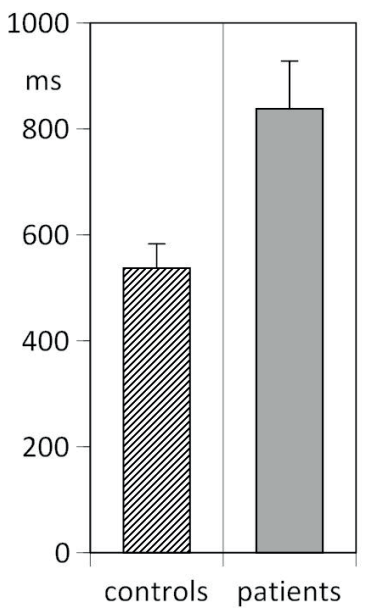

Figure 2. Average pain responsiveness (means, standard errors of means, in ms) for controls (left, $n=94$ ) and patients (right, $n=47$ ). Responsiveness was significantly less in patients ( $t 139=3.30, p=0.001$ )

In addition, $\mathrm{CO}_{2}$ pain responsiveness exhibited a significant correlation with the 32-item odour identification test score (session 1 : $r 94=-0.29, p=0.005$; session 2: $r 94=-0.21, p=0.046$ ) with subjects being more sensitive to $\mathrm{CO}_{2}$ having higher scores in odour identification. Percentile distributions of results for the $\mathrm{CO}_{2}$ pain responsiveness for session 1 are shown in Table 1 . During session 1, eight people showed a consistent ceiling effect meaning that through all 3 trials per session they did not perceive $\mathrm{CO}_{2}$ as painful with 3 of them being patients and another 5 being controls, which is $5.7 \%$ of the entire group tested. In these people data were not used.

When comparing controls and patients, we found - on average
- lower $\mathrm{CO}_{2}$ - pain responsiveness in patients (controls: $\mathrm{M}=537$ $\mathrm{ms}, \mathrm{SD}=448$; patients: $\mathrm{M}=838 \mathrm{~ms}, \mathrm{SD}=619$ ) (Figure 2). This effect was significant ( $\mathrm{t} 139=3.30, \mathrm{p}=0.001)$ although patients were also significantly older than controls (controls: $M=44$ years, $S D=19$; patients: $M=58$ years, $S D=13 ; \mathrm{t} 139=4.37, \mathrm{p}<$ $0.001)$. When only comparing participants older than 50 years, age was no longer significantly different between patients and controls ( $t 79=0.17, \mathrm{p}=0.86$ ) but pain responsiveness was (controls: $\mathrm{M}=612 \mathrm{~ms}, \mathrm{SD}=522 \mathrm{~ms}$; patients: $\mathrm{M}=928 \mathrm{~ms}, \mathrm{SD}=622$ $\mathrm{ms} ; \mathrm{t79}=2.49, \mathrm{p}=0.015)$.

When only investigating the presence of a ceiling effect for $\mathrm{CO}_{2}$ - pain responsiveness, we found that in healthy controls 5 of 100 participants (5\%) exhibited a ceiling effect in all 3 measurements per session, whereas in patients this was the case in 3 of 50 patients (6\%). Although group sizes became relatively small, there was no significant effect of the various causes of olfactory loss (acute infections, head trauma, sinunasal disease, idiopathic; $F[3,43]=1.53, p=0.22$ ) on $\mathrm{CO}_{2}$ pain responsiveness.

\section{Discussion}

This current study had four main findings: (1) Using the new, automated device $\mathrm{CO}_{2}$ pain responsiveness can be measured reliably, (2) $\mathrm{CO}_{2}$ pain responsiveness correlates with olfactory function, (3) as with olfaction, women are more sensitive to $\mathrm{CO}_{2^{\prime}}$ and $\mathrm{CO}_{2}$ - pain responsiveness also correlates with aging, (4) $\mathrm{CO}_{2}$ - pain responsiveness is lower in patients with olfactory loss compared to normosmic, healthy controls.

The most important result from this series of experiments is that the current approach allows to assess $\mathrm{CO}_{2}$ pain responsiveness in a reliable manner. Although other techniques ultimately provide similar results (e.g., $(17,24-27)$; for review see $\left.{ }^{(11)}\right)$ it is important to note that the current approach takes little time and is more or less self-organized by the subject. In addition, costs for producing such a device are low, especially as no dilution of $\mathrm{CO}_{2}$ Is required but $100 \% \mathrm{v} / \mathrm{v} \mathrm{CO}$ is only presented in stimuli of various durations which simplifies stimulus control. The current approach is based on the assumption that - within a certain timeframe - the intranasal trigeminal system acts as an integrator to signal the overall number (mass) of stimulus molecules ${ }^{(17)}$. For a low concentration stimulus to be perceived as strong as a high concentration one, it has to be presented for a longer period of time (e.g., $\left.{ }^{(25)}\right)$.

Indicating its validity, results based on the current approach are related to sex and age, with trigeminal function being highest in young women. This reproduces previous work showing that women respond exhibit a higher sensitivity than men ${ }^{(15,28-30)}$, and older people being less sensitive than younger ones ${ }^{(31-34)}$. In this context, it also seems to be important that the current 
Table 1. Distribution of CO2-pain responsiveness data (in ms) in relation to three age groups (20-40, 41-60 and older than 60 years), separately for patients and controls.

\begin{tabular}{|c|c|c|c|c|c|c|c|}
\hline \multicolumn{2}{|c|}{ age group } & \multicolumn{2}{|c|}{ 20-40 years } & \multicolumn{2}{|c|}{ 41-60 years } & \multicolumn{2}{|c|}{$>60$ years } \\
\hline $\mathbf{N}$ & & controls & patients & controls & patients & controls & patients \\
\hline mean & 0 & 46 & 6 & 23 & 17 & 25 & 24 \\
\hline \multirow[t]{2}{*}{ SEM } & 1 & 466 & 606 & 448 & 790 & 749 & 931 \\
\hline & & 53 & 210 & 87 & 168 & 111 & 121 \\
\hline \multirow[t]{5}{*}{ Percentile } & 10 & 121 & 104 & 134 & 107 & 126 & 173 \\
\hline & 25 & 153 & 139 & 195 & 158 & 331 & 442 \\
\hline & 50 & 283 & 517 & 296 & 596 & 591 & 761 \\
\hline & 75 & 776 & 1018 & 550 & 1454 & 1210 & 1379 \\
\hline & 90 & 957 & & 1244 & 1891 & 1652 & 1911 \\
\hline
\end{tabular}

work also provides first, tentative normative data which allow the rough gauging of trigeminal function on the basis of agerelated values. However, here it has to be kept in mind that the current study is more a feasibility study than a validation study. The currently reported percentiles are likely to change the more subjects will be tested.

It is important to mention that - under the current circumstances $-5 \%$ of the tested population did not perceive $\mathrm{CO}_{2}$ as painful. Thus, future studies should also investigate detection thresholds to overcome the current problem of missing data.

The current results indicate that trigeminal function is reduced in patients with olfactory loss, which is still the case when controlling for age; also the present results are correlated with results from odour identification tests. This relation has already been noted years ago ${ }^{(35)}$, and then this was followed up in some detail ${ }^{(36)}$. The conclusion was that loss of olfactory function leads to changes both in the periphery and the central-nervous processing of trigeminal stimuli, which ultimately leads to a decreased trigeminal sensitivity in patients with acquired loss of function. Because the trigeminal system is involved in the perception of nasal airflow ${ }^{(5,37,38)}$ it is also related to a sensation of a "congested nose". As already mentioned in the Introduction, it has been suggested that nasal airflow perception is related to cooling of the nasal mucosa, and much less so to congestion of the nasal passages ${ }^{(7,8)}\left(\right.$ compare $\left.^{(10)}\right)$. This sensory component of nasal airflow perception could be a reason for the dissatisfaction of patients after septoplasty ${ }^{(39,40)}$. Furthermore, previous studies demonstrated a low correlation between subjective symptoms regarding nasal obstruction, and nasal anatomy ${ }^{(41,42)}$. Thus, the currently introduced, simple technique - provided its wider distribution - may help to shed some light on the above issue as it appears to be suited for the routine investigation of patients, e.g., before and after surgery.

\section{Conclusions}

In conclusion, we have demonstrated that the current approach is a reliable and valid measure of intranasal trigeminal function.

\section{Acknowledgement}

We did not receive any financial help from sources outside the TU Dresden.

\section{Author contributions}

Conceived and designed the experiments: $\mathrm{TH}, \mathrm{KH}$. Performed the experiments: NR, VB, AH, KH. Analysed the data: JL, AU. Wrote the paper: $\mathrm{JL}, \mathrm{TH}$.

\section{Conflict of interest}

The authors have declared that no competing interests exist.

\section{References}

1. von Skramlik E. Über die Lokalisation der Empfindungen bei den niederen Sinnen. Z Sinnesphysiol. 1924; 56: 69.

2. Elsberg CA, Levy I, Brewer ED. The sense of smell VI. The trigeminal effects of odorous substances. Bull Neurol Inst NY. 1935; 4: 270-285.

3. Doty RL, Brugger WPE, Jurs PC, Orndorff MA, Snyder PJ, Lowry LD. Intranasal trigeminal stimulation from odorous volatiles: psychometric responses from anosmic and normal humans. Physiol Behav. 1978; 20: 175-185. 4. Cometto-Muniz JE, Hernandez SM. Odorous and pungent attributes of mixed and unmixed odorants. Percept Psychophys. 1990; 47: 391-399.

5. Eccles R, Jones AS. The effect of menthol on 
nasal resistance to air flow. J Laryngol Oto .1983; 97: 705-709.

6. Lindemann J, Tsakiropoulou E, Scheithauer MO, Konstantinidis I, Wiesmiller KM. Impact of menthol inhalation on nasal mucosa temperature and nasal patency. Am J Rhinol. 2008; 22: 402-405.

7. Zhao K, Blaker K, Luo Y, Bryant B, Jiang J. Perceiving nasal patency through mucosal cooling rather than air temperature or nasal resistance. PLoS ONE. 2011; 6: e24618.

8. Zhao K, Jiang J, Blacker K, Lyman B, Dalton P, Cowart BJ, Pribitkin EA. Regional peak mucosal cooling predicts the perception of nasal patency. Laryngoscope. 2014; 124: 589-595.

9. Scheibe M, Schulze S, Mueller CA, Schuster B, Hummel T. Intranasal trigeminal sensitivity: measurements before and after nasal surgery. Eur Arch Otorhinolaryngol. 2014; 271: 87-92.

10. Schriever $V$, Hummel T. Subjective changes in nasal patency after chewing a mentholcontaining gum in patients with olfactory loss. Acta Oto-Laryngologica. 2015; 135 254-257.

11. Hummel T. Assessment of intranasal trigeminal function. Int J Psychophysiol. 2000; 36: 147-155.

12. Fröhlich R. Ueber einige Modificationen des Geruchsinnes. Akad Wiss Wien, math-nat CL 1851; 6: 322-328.

13. Kobal G, Van Toller S, Hummel T. Is there directional smelling? Experientia1989; 45: 130-132.

14. Frasnelli J, Hummel T. Intranasal trigeminal thresholds in healthy subjects. Environ Toxicol Pharmacol. 2005; 19: 575-580.

15. Hummel T, Mohammadian P, March R, Kobal G, Lotsch J. Pain in the trigeminal system: irritation of the nasal mucosa using short- and long-lasting stimuli. Int J Psychophysiol. 2003; 47: 147-158.

16. Negoias S, Aszmann O, Croy I, Humme T. Localization of Odors Can Be Learned. Chem Senses. 2013; 38: 553-562.

17. Cometto-Muniz E, Cain WS. Temporal integration of pungency. Chem Senses. 1984; 8: 315-327.

18. Hummel T, Sekinger B, Wolf SR, Pauli E, Kobal G. 'Sniffin' sticks': olfactory performance assessed by the combined testing of odor identification, odor discrimination and olfactory threshold. Chem Senses. 1997; 22: 39-52.

19. Haehner A, Mayer AM, Landis BN, et al. High test-retest reliability of the extended version of the "Sniffin' Sticks" test. Chem Senses. 2009; 34: 705-711.

20. Sorokowska A, Albrecht E, Haehner A,
Hummel T. Extended version of the "Sniffin" Sticks" identification test: test-retest reliability and validity. J Neurosci Methods. 2015; 243:111-114.

21. Hummel T, Kobal G, Gudziol H, Mackay-Sim A. Normative data for the "Sniffin' Sticks" including tests of odor identification, odor discrimination, and olfactory thresholds: an upgrade based on a group of more than 3,000 subjects. Eur Arch Otorhinolaryngol. 2007; 264: 237-243.

22. Croy I, Lange K, Krone F, Negoias S, Seo HS, Hummel T. Comparison between odor thresholds for phenyl ethyl alcohol and butanol. Chem Senses. 2009; 34: 523-527.

23. Hummel T, Landis BN, Huttenbrink KB. Smell and taste disorders. GMS Curr Top Otorhinolaryngol Head Neck Sur 2011; 10: Doc04.

24. Naka A, Wolf A, Renner B, Mueller CA. A novel device for the clinical assessment of intranasal trigeminal sensitivity. Ann Otol Rhinol Laryngol. 2014; 123: 428-433.

25. Frasnelli J, Hummel T, Berg J, Huang G, Doty RL. Intranasal localizability of odorants: influence of stimulus volume. Chem Senses 2011; 36: 405-410.

26. 26. Wise JB, Moonis G, Mirza N. Magnetic resonance imaging findings in the evaluation of traumatic anosmia. Ann Otol Rhinol Laryngol. 2006; 115: 124-127.

27. Kobal G. Elektrophysiologische Untersuchungen des menschlichen Geruchssinns. Stuttgart: Thieme Verlag, 1981.

28. Krone D, Mannel M, Pauli E, Hummel T. Qualitative and quantitative olfactometric evaluation of different concentrations of ethanol peppermint oil solutions. Phytother Res. 2001; 15: 135-138.

29. Cometto-Muniz E, Noriega G. Gender differences in the perception of pungency. Physiol Behav. 1985; 34: 385-389.

30. Frasnelli J, Hummel T. Age related decline of intranasal trigeminal sensitivity: Is it a peripheral event? Brain Res. 2003; 987: 201 206

31. Hummel T, Heilmann S, Murphy C. Agerelated changes of chemosensory functions. In: Rouby C, Schaal B, Dubois D, Gervais R, Holley A, eds. Olfaction, taste and cognition. New York: Cambridge University Press, 2002: 441-456.

32. Hummel T, Futschik T, Frasnelli J, Huttenbrink KB. Effects of olfactory function, age, and gender on trigeminally mediated sensations: a study based on the lateralization of chemosensory stimuli. Toxicol Lett. 2003; 140-141: 273-280

33. Wysocki CJ, Cowart BJ, Radil T. Nasal trigem- inal chemosensitivity across the adult life span. Percept Psychophys. 2003; 65: 115122

34. Stuck BA, Frey S, Freiburg C, Hormann K, Zahnert T, Hummel T. Chemosensory event-related potentials in relation to side of stimulation, age, sex, and stimulus concentration. Clin Neurophysiol. 2006; 117: 1367-1375.

35. Kobal G, Hummel C. Cerebral chemosensory evoked potentials elicited by chemical stimulation of the human olfactory and respiratory nasal mucosa. Electroenceph Clin Neurophysiol. 1988; 71: 241-250.

36. Frasnelli J, Schuster B, Hummel T. Interactions between olfaction and the trigeminal system: what can be learned from olfactory loss. Cereb Cortex. 2007; 17: 2268-2275.

37. Tsubone H. Nasal 'flow' receptors of the rat. Resp Physiol. 1989; 75: 51-64.

38. Tsubone H. Nasal 'pressure' receptors. Nippon juigaku zasshi. 1990; 52: 225-232.

39. Konstantinidis I, Triaridis S, Triaridis A, Petropoulos I, Karagiannidis K, Kontzoglou G. How do children with adenoid hypertrophy smell and taste? Clinical assessment of olfactory function pre- and post-adenoidectomy. Int J Pediatr Otorhinolaryngol. 2005; 69: 1343-1349.

40. Illum P. Septoplasty and compensatory inferior turbinate hypertrophy: long-term results after randomized turbinoplasty. Eur Arch Otorhinolaryngol. 1997; 254 Suppl 1: S89-92.

41. Dinis PB HH-. Septoplasty: long-term evaluation of results. Am J Otolaryngol. 2002; 23: 85-90.

42. Courtiss EH, Goldwyn RM. The effects of nasal surgery on airflow. Plast Reconstr Surg. 1983; 72: 9-21.

Thomas Hummel, M.D.

Smell \& Taste Clinic

Department of Otorhinolaryngology

TU Dresden

Fetscherstrasse 74

01307 Dresden

Germany

Tel: +49-351-458 4189

Fax: +49-351-458-4326

E-mail: thummel@mail.zih.tu-resden. de 